\title{
Study of the Surface Resistance of Niobium Sputter-Coated Copper Cavities
}

\author{
C. Benvenuti, S. Calatroni, I. E. Campisi, ${ }^{a}$ P. Darriulat, M. A. Peck, R. Russo and A.-M. Valente \\ CERN, 1211 Geneva 23, Switzerland
}

\begin{abstract}
A systematic study of the superconducting properties of Niobium films deposited on the inner wall of Copper radiofrequency cavities is presented. Films are grown by sputtering with different discharge gases (Xe, $\mathrm{Kr}$, $\mathrm{Ar}$ and Ar/Ne mixtures) on substrates prepared under different conditions. The measured quantities include the surface resistance at $1.5 \mathrm{GHz}$, the critical temperature and the penetration depth. The surface resistance is analyzed in terms of its dependence on temperature, RF field and the density of trapped fluxons. Once allowance for electron scattering is made by means of a single mean free path parameter, good agreement with BCS theory is observed.

The residual resistance is observed to be essentially noncorrelated with the superconducting properties, although influenced by specific coating conditions. In occasions, very low residual resistances, in the nano-ohm range, have been maintained over a broad range of $R F$ field, indicating the absence of fundamental limitations specific to the film technology in practical applications.
\end{abstract}

\section{INTRODUCTION}

The aim of the present study [1] is to improve the understanding of the mechanism of RF losses in niobium coated cavities [2], such as those successfully employed in LEP2 at CERN [3]. For this purpose, more than 100 coatings have been performed and characterized. Reasons of manageability suggest the use of $1.5 \mathrm{GHz}$ copper resonators, operated in the $\mathrm{TM}_{010}$ mode. The coating parameters explored include among others the technology for manufacturing the resonators (spinning, hydroforming or electroforming), the type of gas used for sputtering (Xe, $\mathrm{Kr}$, $\mathrm{Ar}, \mathrm{Ne}$ or Ar-Ne mixtures in various proportions) and the presence or not of an oxidized layer on the copper surface. Some of the films have also been loaded with hydrogen before the final exposure to air, and outgassed after the first RF measurement.

All the resonators have been characterized by measuring their surface resistance as a function of the RF field, at different temperatures and in presence of various amounts of trapped magnetic flux. Penetration depth, first magnetic penetration field (obtained by means of a superconducting coil acting at the equator of the cavity perpendicularly to the surface) and $\mathrm{T}_{c}$ complete the array of measurements performed. The novelty of the present study lies in the accuracy of the data analysis performed on all the

\footnotetext{
${ }^{a}$ Present address: TJNAF, Newport News, Virginia, 23606, USA
} Manuscript received September 15, 1998 measurements, together with unprecedented statistics. No systematic attempts have been done for obtaining high accelerating fields, this being more a technological problem than a fundamental one.

A few cavities have been cut in order to analyze in more detail the film properties. The characterizations included RRR and $T_{c}$ measurements (with and without the copper substrate), complete XRD analyses, SEM and TEM observations, AES and SIMS elemental composition studies.

A complete summary of these results may be found in [1]. The main conclusion is that all the variables that influence the BCS surface resistance have no reproducible effect on the residual resistance. On the other hand, a few variables have been observed to definitely have an influence only on the residual resistance and not on other superconducting properties. In this paper, we will concentrate on this latter aspect, and on possible strategies to reduce the residual resistance.

\section{THE RESIDUAL RESISTANCE}

The surface resistance is usually divided into the sum of three terms, $R_{s}=R_{B C S}+R_{f l}+R_{\text {res. }}$. The first term, the $B C S$ surface resistance, is described at zero $\mathrm{RF}$ field only by firstorder theoretical models [4], which predict a minimum at an electron mean free path $\ell$ close to the BCS coherence length $\xi_{0}$. The RF field dependence can be factorized empirically as the product of the zero field value times a temperature independent term.

No detailed theoretical calculations exist to describe the effect of a low trapped magnetic flux on the surface resistance and its RF field dependence, accounted for by the second term, the fluxon-induced losses. This term has been shown [1] to have a similar but much stronger dependence on $\ell$ than the first term. Both present however a minimum for a value of $\ell$ which is the same within experimental error.

The third term, the residual resistance, usually attributed to impurities and defects in the superconductor [5], can often be written as $\mathrm{R}_{\mathrm{res}}{ }^{0}+\mathrm{R}_{\mathrm{res}}{ }^{1} \mathrm{H}_{\mathrm{RF}}$, in particular at low $\mathrm{RF}$ field. The zero $R F$ field value $R_{\text {res }}{ }^{0}$ may vary for different cavities from values as small as $1 \mathrm{n} \Omega$ and up to $100 \mathrm{n} \Omega$, and a similar range of variations has been observed for its $R F$ field dependence $R_{r e s}{ }^{1}$. No variations in $R_{B C S}, R_{f l}$ or other superconducting properties have been observed in correlation with these changes in residual resistance. It has however been found that the way of manufacturing the substrate, the passivation layer on the copper surface, and a loading of the film with hydrogen have all a definite effect on the residual resistance. 


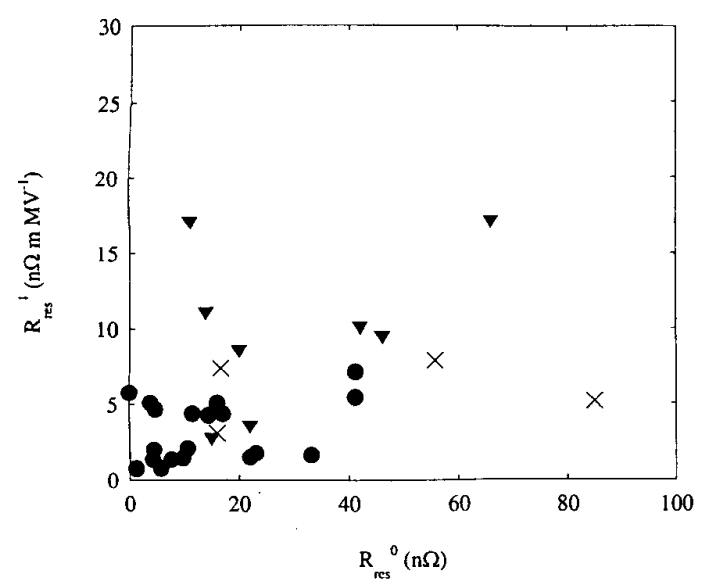

Fig. 1. Rate of increase of the residual resistance with $\mathrm{RF}$ field as a function of the residual resistance at zero RF field. Spun cavities are indicated by dots, hydroformed by triangles and electroformed by crosses.

\section{A. Roughness Effect}

Niobium films deposited according to the procedure employed for LEP2 cavities are defined as standard coatings. The coating parameters have been of course adapted to the geometry of $1.5 \mathrm{GHz}$ cavities, but the essential features such as substrate preparation, bakeout and coating temperatures and sputter gas employed (argon) have been kept unchanged. Nineteen standard coatings of $1.5 \mu \mathrm{m}$ thickness have been done on cavities produced by spinning, nine on hydroformed cavities and four on prototype electroformed cavities. Their zero RF field residual resistance and the overall rate of increase with RF field are shown in Fig. 1. Spun cavities are found to have better values of residual resistance than hydroformed cavities, and a lower RF field dependence. Extensive material analyses have been carried out on the copper resonators after forming, to identify the reasons for this behavior. The major differences are the average roughness $(0.2 \mu \mathrm{m}$ for spun cavities, $1 \mu \mathrm{m}$ for hydroformed ones), and the shape and dimension of copper grains $(100 \mu \mathrm{m} \times 10 \mu \mathrm{m}$ elongated for spun cavities and $100 \mu \mathrm{m}$ equiaxed for hydroformed ones). No clear relationship has however been established between the metallurgical properties of copper and those of the niobium film deposited on it. Roughness is known to influence the grain growth and the structure of the film, because of shadowing of the niobium atoms arriving onto the substrate [6]. It may also simply result in small regions of copper left uncoated or coated with a layer too thin to completely shield the radiofrequency.

These considerations lead naturally to the search for substrates with the lowest achievable roughness. A possible solution explored consists in producing cavities by electroplating copper on highly polished mandrels, made by either glass or aluminum. This procedure is not optimized yet, and it still leads to the formation of defects in the copper matrix. The first results are however encouraging, as illustrated in Fig. 1. The high residual resistance obtained in two cases was the result of film peel-off at defects like those mentioned above, and thus not intrinsic.

\section{B. The Niobium-Copper Interface}

In the standard LEP2 procedure, the cavity surface is passivated at the end of the chemical preparation, and then exposed to (dust-free) air. An oxide layer is thus present on the copper surface, which remains underneath the niobium coating. A special two-cathode coating system, which allows in-situ sputter-etching of the oxide before coating, has been employed to coat several cavities. Oxide removal from the substrate is usually considered common good practice in thin film technology. When applied to standard niobium films coated on copper by keeping all other conditions unchanged, higher RRR (about 30) have been obtained compared to standard films with an oxide layer (about 10). These values are confirmed by the values of $\ell$ derived from penetration depth measurements. The texture of these films measured by $\mathrm{X}$-ray diffraction is also different. Standard films grow preferentially with the (110) plane parallel to the surface, while for oxide-free films the (211) and (200) planes are also equally probable. The lattice parameter in the direction normal to the surface is slightly smaller for films without oxide, and associated with the difference in critical temperature $(\sim 9.35 \mathrm{~K}$ for oxide-free films instead of $\sim 9.50 \mathrm{~K}$ ), this may imply a lower thermal stress during cooldown.

It has been found that cavities coated according to the two different procedures have also slight differences in the residual resistance. In Fig. 2 are illustrated the residual resistance $R_{\text {res }}{ }^{0}$ and its RF field dependence $R_{\text {res }}{ }^{1}$ for thirty-six spun cavities. These have been coated using argon, krypton or xenon as sputter gas, which all give similar results in terms of all the other superconducting properties. All cavities with

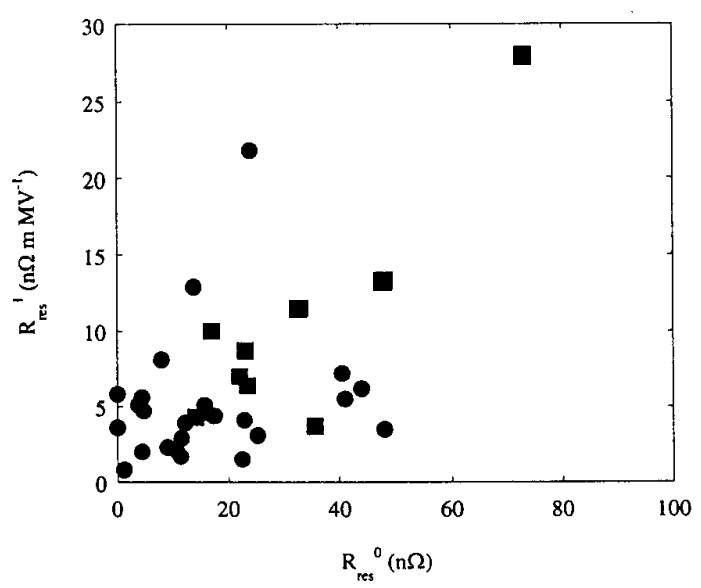

Fig. 2. Residual resistance $R_{\text {res }}{ }^{0}$ and its $R F$ field dependence $R_{\text {res }}{ }^{\prime}$ for standard cavities (dots) and for cavities without oxide interface (squares). 


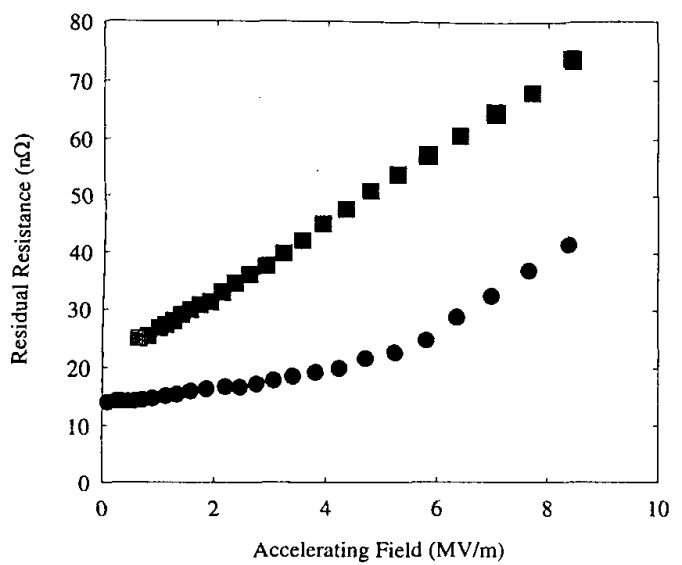

Fig. 3. Dependence of the residual resistance on the accelerating RF field for two particular cavities, one with oxide interface (dots) and the other without oxide interface (squares).

a residual resistance higher than $100 \mathrm{n} \Omega$ or a slope coefficient higher than $50 \mathrm{n} \Omega \mathrm{m} \mathrm{MV}^{-1}$ have been arbitrarily excluded from the analysis. It is apparent that both the residual resistance and the RF field dependence are higher for cavities coated without oxide. The average value of $R_{\text {res }}{ }^{0}$ is $31 \pm 6 \mathrm{n} \Omega$ for oxide-free cavities, and $17 \pm 3 \mathrm{n} \Omega$ for cavities with oxide. The average value of $R_{\text {res }}{ }^{1}$ is $10 \pm 2 \mathrm{n} \Omega \mathrm{m} \mathrm{MV}^{-1}$ for oxide-free cavities, and $5 \pm 1 \mathrm{n} \Omega \mathrm{m} \mathrm{MV}^{-1}$ for cavities with oxide.

The detailed dependence of the residual resistance on the RF field, for two particular cavities coated according to the two different procedures, is shown in Fig. 3. These two cavities have been chosen because their difference is very pronounced, although a qualitatively similar behavior is displayed by all the cavities measured. Cavities coated without an oxide layer at the niobium copper interface have a linear dependence over the entire RF field range. Cavities coated with an oxide layer have instead a kink in the data, which separates two regions where the RF field dependence is linear.

To quantify this difference, the data have been tentatively fit up to the field emission threshold as $\mathrm{R}_{\mathrm{res}}{ }^{0}+\mathrm{R}_{\mathrm{res}}{ }^{1} \mathrm{H}_{\mathrm{RF}}+\mathrm{R}_{\mathrm{res}}{ }^{2}\left(\mathrm{H}_{\mathrm{RF}}-\mathrm{H}_{\mathrm{k}}\right)$, the third term being valid only for $\mathrm{H}_{\mathrm{RF}}>\mathrm{H}_{\mathrm{k}}$. It is found that most (about $85 \%$ ) of the coatings without an oxide layer can indeed be described by a single fit of slope $\mathrm{R}_{\text {res }}{ }^{1}$ over the entire RF field range. Most (about $90 \%$ ) of the coatings with an oxide layer must be described by two separate fits, the slope in the high field region being always bigger than the slope in the low field region. Although the RF field dependence is on average smaller below the threshold $\mathrm{H}_{\mathrm{k}}$ for cavities with an oxide layer, this difference disappears above the threshold.

It is interesting to note that the field threshold, when present, has a value which is close to the one measured for fluxon induced losses [1].

\section{Effect of Hydrogen}

It is well known that the residual resistance of cavities made of high-purity bulk niobium is influenced by the hydrogen content in the metal [7]. Hydrogen may in fact under certain conditions segregate on the cavity surface to form hydrides, thus leading to higher residual resistances. Impurities, although reducing the RRR, can act as trapping centers for hydrogen and reduce this effect. A similar phenomenon can be expected in niobium films. It is quite possible that the hydrogen present in high-purity films, such as standard coatings without oxide layer, acts differently compared to films where the oxide layer is present. The total quantity of hydrogen contained in the films is estimated in the range between $0.1 \%$ and $1 \%$, and is in the process of being accurately measured.

To verify that the effect of hydrogen depends on purity, two standard coatings with and without the oxide interface have been loaded with about $1.5 \%$ (atomic) of hydrogen, before the final exposure of the film surface to air. The
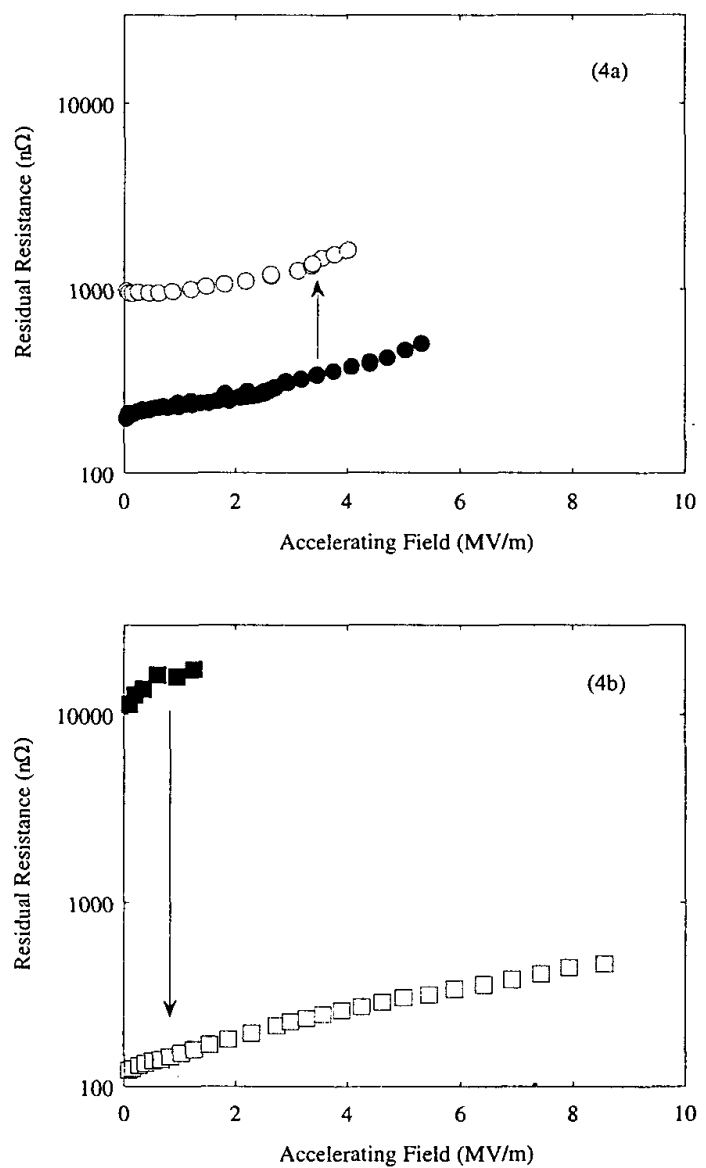

Fig. 4. Residual resistance for cavities loaded with hydrogen, with oxide (4a) and without oxide (4b). Full symbols indicate the first RF measurement of loaded films, empty symbols indicate the values after outgassing. 
results are shown in Fig. 4. The cavity with an oxide layer has a residual resistance that, although higher than average, is still much lower than for the cavity without oxide. The residual resistance of the latter is extremely high, but a subsequent outgassing of the cavity at $300^{\circ} \mathrm{C}$ for about 12 hours restores a reasonable value of residual resistance. During the outgassing, about half of the hydrogen that was put into the film is released. A similar outgassing cycle performed on the cavity with oxide led however to an increase of residual resistance. While these data are preliminary, the hypothesis that hydrogen can deteriorate the residual resistance of very clean films is confirmed by this test. A more detailed study is under way to understand whether the hydrogen naturally present in the films contributes a major part of their residual resistance.

\section{CONCLUSION}

The residual resistance of niobium film cavities appears to be uncorrelated with the measured superconducting properties. Other parameters, such as the surface roughness or the hydrogen content (depending on the total purity of the film) are observed to exert an influence on it. A possible way to reduce the roughness of the substrate has been explored, and others could be envisaged, such as a different chemical polishing of ordinary cavities. Although the exact contribution of hydride precipitation to the residual resistance remains to be measured, it has been shown that hydrogen loading definitely increases the residual resistance of cleaner films. Decreasing the hydrogen content of films, by applying either a suitable gettering underlayer or a thorough outgassing of all the coating components, is under study.

\section{REFERENCES}

[1] C. Benvenuti, S. Calatroni, I. E. Campisi, P. Darriulat, M. A. Peck, R. Russo, A.-M. Valente, Physica $C$, in press.

[2] C. Benvenuti, Part. Accel., vol. 40, p. 43, 1992.

[3] D. Boussard, Proc. of the Sth EPAC, S. Myers, Ed. Bristol: IOP, 1996, p. 187.

[4] J. Halbritter, Z. Phys., vol. 266, p. 209, 1974

[5] J. Halbritter, J. Appl. Phys., vol. 68, n. 12, p. 6315, 1990.

[6] G. S. Bales and A Zangwill, J. Vac. Sci. Technol., vol. A9, n. 1, p. 145, 1991.

[7] B. Bonin and R. W. Roeth, Proc. of the 5th Workshop on RF Superconductivity, D. Proch, Ed. Hamburg: DESY, 1991, p. 210. 